\title{
Radiographic assessment of the percutaneous Bianchi system technique for treatment of hallux valgus
}

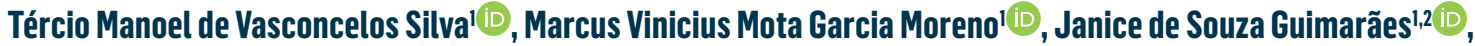 \\ Túlio Eduardo Vieira Marçal' ${ }^{\mathbb{D}}$, Thiago Batista Faleiro ${ }^{2,3}{ }^{\mathbb{D}}$, Marilton Jorge Torres Gomes ${ }^{1}$ (D) Michele Risi $^{4}$ (D) \\ 1. Hospital COT - Hospital Ortopédico e Traumatológico, Salvador, BA, Brazil. \\ 2. Universidade Federal da Bahia, Salvador, BA, Brazil. \\ 3. Hospital Santo Antônio, Obras Sociais Irmã Dulce, Salvador, BA, Brazil. \\ 4. Associazione professionale PBS. Casa Di Cura Villa Donatello, Firenze, Italy.
}

\section{Abstract}

Objective: To present initial radiographic results of surgical correction of the hallux valgus angle (HVA) and the intermetatarsal angle (IMA) using the percutaneous Bianchi system (PBS) technique.

Methods: Seventeen patients with moderate to severe hallux valgus (HV) were exclusively treated with the PBS technique and assessed radiographically preoperatively and during the postoperative period, from January 2019 to January 2020. The degree of deformity correction was recorded, based on the HVA and the IMA. Stata (v. 14.0) software was used for statistical analyses. Pre-surgical and post-surgical mean HVA and IMA were compared using Student's $t$ test for paired samples and the McNemar test was used to compare HVA and IMA categories. Statistical significance was set at 5\% and 95\% confidence intervals were estimated.

Results: Both HVA and IMA were reduced significantly during the assessment period. Mean radiographic correction of the HVA was $15.1^{\circ}$ and mean radiographic correction of the IMA was 7.3 .

Conclusions: According to the results presented, use of the PBS technique achieved adequate correction of the radiographic parameters of the patients who underwent the treatment as proposed, although it is necessary to conduct additional studies with longer follow-up to achieve a higher recommendation level.

Level of Evidence IV; Therapeutic Studies; Case Series.

Keywords: Hallux valgus; Osteotomy; Metatarsal bones; Metatarsophalangeal joint.

\section{Introduction}

Hallux valgus (HV) is a common disorder of the forefoot ${ }^{(1)}$ that was first described in a scientific report published by Volkmann in 1856. However, it was left to Carl Hueter apud Nery ${ }^{(2)}$ to define the deformity in 1871, which he characterized as a lateral deviation (in valgus) of the hallux, combined with a medial deviation (in varus) of the head of the first metatarsal bone(3). Epidemiologically, it presents more frequently among females, aged from 40 to 60 years, with ratios reported in studies varying up to 15 women for each man affected, and it is generally bilateral ${ }^{(2)}$. There is a direct association with the type of footwear worn and with a variety of intrinsic ana- tomic and genetic factors and even with other systemic conditions, with regard to determinants of its functional severity and progression ${ }^{(1)}$.

During the 2010s, there was growing interest in use of minimally invasive surgery to correct HV. Reverdin-Isham pioneered the study of percutaneous surgery for treatment of $\mathrm{HV}$, proposing procedures based on an incomplete oblique intraarticular osteotomy of the head of the first metatarsal. With improvements to the percutaneous techniques proposed by Bösch et al. (1) and, later, by Giannini et al.(4) and Magnan et al. ${ }^{(5)}$, a distal and transverse percutaneous osteotomy of the first metatarsal was developed for correction of mild and moderate HV.
Study performed at the Hospital COT - Hospital Ortopédico e Traumatológico, Salvador, BA, Brazil.

Correspondence: Tércio Manoel de Vasconcelos Silva. Rua João das Botas, 28, Canela - 40110-160, Salvador, BA, Brazil. E-mail: terciomvs@gmail.com Conflicts of interest: none. Source of funding: none. Date received: April 28, 2021. Date accepted: July 16, 2021. Online: August 31, 2021.

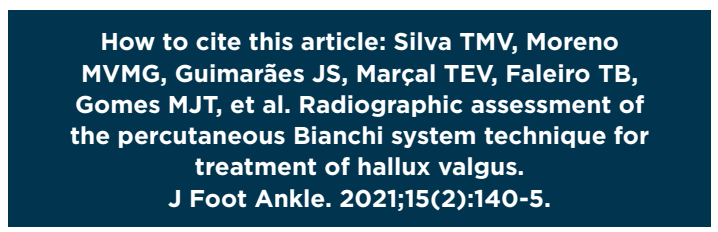
MVMG, Guimarães JS, Marcal TEV, Faleiro TB, Gomes MJT, et al. Radiographic assessment of treatment of hallux valgus. J Foot Ankle. 2021;15(2):140-5. 
Countless procedures, with different surgical approaches and fixation hardware(6) are described in the global literature for surgical treatment of this condition. The share the common objectives of correcting the inherent deformities that cause $\mathrm{HV}$, improving pain and foot function, achieving low rates of relapse ${ }^{(1-3,6-9)}$, reducing operating time, and enabling the procedure to be performed bilaterally in a single operating session $^{(8,10)}$. In this context, the objective of the present study is to present the radiographic results of surgical correction of moderate and severe hallux valgus, using the percutaneous Bianchi system (PBS), which is a technique that employs a complete, extra-articular, nonlinear, distal osteotomy of the first metatarsal(6).

\section{Methods}

This study was approved by the Human Research Ethics Committee. This was a retrospective observational study with a case series design, conducted at a private orthopedics and traumatology clinic.

After each patient had read and signed the free and informed consent form (TCLE) agreeing to participate in the study, data collection was begun. Patients included in this study were all managed by the same surgical team, comprising a lead surgeon and an assistant surgeon, both of whom were orthopedists and specialists in foot and ankle surgery, and all patients exclusively underwent surgical treatment with the percutaneous PBS technique. Preoperative and postoperative radiographic examinations were analyzed for a 12-month period spanning January 2019 to January 2020, in addition to data collection via electronic medical records.

Inclusion criteria were as follows: patients over the age of 18 years, with moderate to severe HV, subjected to the PBS technique only, with persistent pain and deformities, which had not improved in response to conservative measures (changing footwear, physiotherapy, and others) for a minimum of 6 months. The exclusion criteria were: patients who had rigidity of the first metatarsophalangeal joint, rheumatoid arthritis or other inflammatory conditions, patients with diabetes, neurological disorders, hypermobility of the first tarsometatarsal joint, patients who needed an Akin osteotomy for correction of interphalangeal hallux valgus, and patients who had previously undergone some type of surgical procedure on the hallux.

$X$-ray images were acquired using the technique described by Tanaka et al.(11), in which the patients are positioned standing on the film, obtaining a weightbearing anteroposterior view. These images were used to measure the hallux valgus angle (HVA) and the intermetatarsal angle (IMA), measured between the 1st and 2nd metatarsals, as recommended by the American Orthopedic Foot and Ankle Society (AOFAS) ${ }^{(4)}$. The HVA is the angle formed by the axis of the proximal phalanx and the axis of the first metatarsal. The IMA is the angle formed by the axes of the first and second metatarsals.

All of the procedures and measurements were performed by the lead surgeon, using the standard PBS operating technique. It should be noted that all patients underwent the same postoperative protocol.
With the aid of Stata (v. 14.0) software, statistical analyses were conducted with descriptive tests, such as calculation of means and standard deviations, to describe the study population. Mean HVA and IMA before and after execution of the PBS technique were compared using Student's $t$ test for paired samples, while Student's $t$ test for independent samples was used to analyze the effects of age and left vs. right foot on reduction of measurements, with the objective of comparing the reduction in mean angles in different age ranges and different side feet (mean differences). The hypothesis of normality was confirmed using the Komogorov-Smirnov test. The McNemar test was used to compare HVA and IMA categories, before and after surgery. The level of statistical significance adopted for analysis was 5\% and 95\% confidence intervals were estimated.

\section{Operating technique}

With the patient supine, under spinal anesthesia or peripheral block, without a pneumatic tourniquet, the foot was positioned around $20 \mathrm{~cm}$ beyond the edge of the operating table.

A Beaver scalpel blade was used to make an approximately $2 \mathrm{~mm}$ incision in the dorsal skin, lateral of the first metatarsophalangeal joint. This incision was used to perform a partial capsulotomy and tenotomy of the hallux adductor tendon, to enable displacement of the head of the first metatarsal and, as a result, varus reduction of the hallux.

A second incision, of $4-5 \mathrm{~mm}$, was then made in the skin slightly proximal and plantar of the head of the metatarsal, on the medial side. Capsulotomy of the first metatarsophalangeal joint of the subjacent bone was then conducted, using a "windshield wiper" action( ${ }^{(8)}$ and, via the same approach, a medial exostectomy was conducted using a Wedge burr ( $4.1 \mathrm{x}$ $13 \mathrm{~mm}$ ). The exostectomy produces a toothpaste-like mixture consisting of bone residues and blood, which was eliminated via the incision by manual expression ${ }^{(8)}$. It should be emphasized that all of these stages are performed with radioscopic control. The same medial approach was then used to conduct the extra-articular osteotomy of the first metatarsal, using a straight Shannon burr $(2 \times 12 \mathrm{~mm})$, with dorsal subcapital and slightly oblique alignment in relation to the longitudinal/coronal axis of the first metatarsal(6) taking care not to complete the first cut, to maintain lateral cortical bone.

Later, the dorsal cortical was osteotomized with a distal-to-proximal action in the sagittal plane, enabling the head of the first metatarsal to be slightly shortened and displaced into a slightly more plantar position. In a final action, the lateral cortical bone was severed with a slightly more oblique cut in the coronal plane, leaving a small step in the lateral border, which helps to stabilize the head of the first metatarsal, maintaining the correction. It is important to point out that the osteotomy can be displaced by up to $90 \%$ of the diameter of the diaphysis of the metatarsal, recentralizing the sesamoids and realigning the first $\operatorname{ray}^{(8)}$. No osteosynthesis materials were used. During exostectomy and osteotomy, the bone was irrigated with saline solution to reduce the risk of thermal necrosis ${ }^{(6)}$. 
After the desired correction had been achieved, an appropriate dressing was applied, consisting of protective bandaging around the hallux which, when correctly positioned, avoids over-correction. The dressing was changed 3 weeks after the surgical procedure and kept in place for a total of 6 weeks, or until formation of signs of consolidation were visible on $\mathrm{X}$-rays ${ }^{(8)}$. Patients were permitted to walk immediately, from the first postoperative day onwards, depending on tolerance, wearing appropriate footwear with a flat and rigid sole ${ }^{(5)}$

\section{Results}

\section{Profile of participants and assessment of the PBS technique}

A total of 19 patients with moderate to severe HV were selected for the study, two of whom were excluded because they underwent osteotomy of the proximal phalanx of the hallux (Akin osteotomy). All of the 17 patients included were women, with a mean age of $51.1 \pm 13.5$ years, ranging from 22 to 66 years. Seven of them were over the age of $60(41.2 \%)$. In terms of laterality, nine underwent surgery on the right foot and eight on the left. There were no cases of bilateral surgery (Table 1).

The radiographic analysis was conducted using preoperative images and images from 12 months after surgery. Standard weightbearing anteroposterior and lateral X-rays were used. The HVA and IMA were analyzed. The HVA was categorized as mild $\left(15-20^{\circ}\right)$, moderate $\left(21-39^{\circ}\right)$, or severe $\left(\geq 40^{\circ}\right)$ and the IMA was categorized as mild $\left(9-11^{\circ}\right)$, moderate $\left(12-17^{\circ}\right)$, or severe $\left(\geq 18^{\circ}\right)^{(4)}$ (Figure 1).

On the basis of their HVA, $76.4 \%$ of cases were classified as severe preoperatively. At 12 postoperative months, all patients had HVA measurements less than $15^{\circ}$, which was a statistically significant difference. The same was observed in relation to the IMA, for which $76.4 \%$ of the patients were clas-

Table 1. Characteristics at the initial assessment of patients with hallux valgus treated with the PBS technique

\begin{tabular}{lc}
\hline Characteristics & Statistic \\
\hline Number of subjects & 17 patients \\
Sex & $17(100 \%)$ \\
Female & \\
Age & $51.1 \pm 13.5$ years \\
Mean \pm SD & $22-66$ years \\
Minimum - Maximum & \\
Age group & $10(58.8 \%)$ \\
$<60$ years & $7(41.2 \%)$ \\
60 years or over & \\
Foot operated (laterality) & $9(52.9 \%)$ \\
Right & $8(47.1 \%)$ \\
Left & $0(0 \%)$ \\
Bilateral &
\end{tabular}

sified as moderate. After 12 months, sixteen patients had IMA measurements less than $9^{\circ}$ and just one patient had an IMA of $9^{\circ}$, classified as mild (Table 2 ). A reduction in the degree of hallux valgus was observed after the surgical procedures.

Comparing the HVA measurements before the intervention and 12 months afterwards, a statistically significant reduction was observed after use of the PBS technique $(p<0.001)$ (Figure 2). The patients exhibited mean scores of $22.9 \pm 4.1$ preoperatively, which reduced to $7.8 \pm 2.7$ during the postoperative period, i.e., there was a mean reduction of $15.1^{\circ}$ (95\% Cl: 13.2 to 17.0 ). A similar result was observed in the analysis of IMA measurements, in which the mean reduction was $7.3^{\circ}$ ( $95 \% \mathrm{Cl}: 5.9$ to 8.7 ), which is a statistically significant difference $(p<0.001)$. The mean preoperative angle was $13 \pm$ 2.2 , reducing to $5.7 \pm 1.5$ after surgery (Table 3 ). Figure 3 illustrates HV before and 12 months after the surgical procedure.
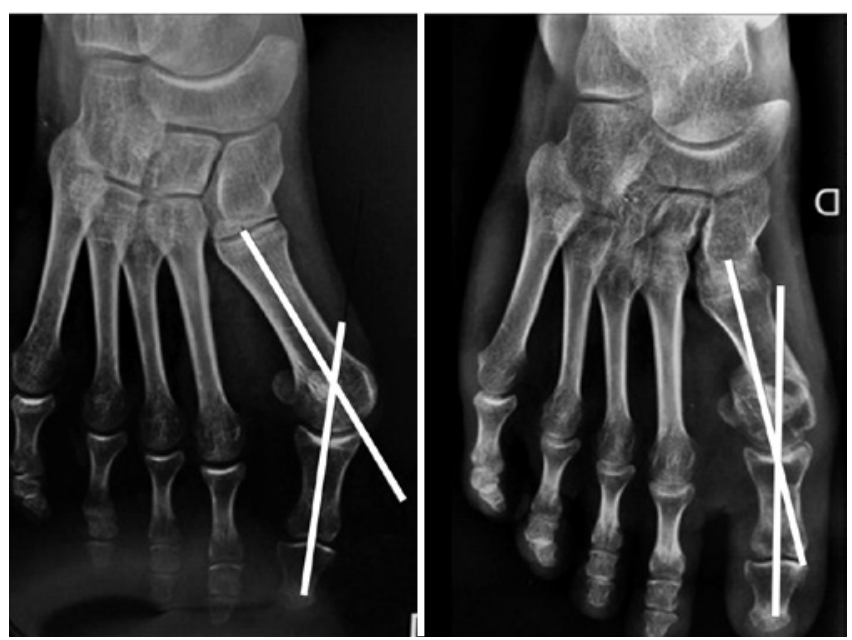

Figure 1. Anteroposterior $\mathrm{X}$-ray showing correction of the hallux valgus angle - Preoperative (left); and 12 months after surgery (right).

Table 2. Comparison of valgus categories based on the hallux valgus angle and the angle between the first and second metatarsals, before and after intervention, in patients with hallux valgus treated with the PBS technique

\begin{tabular}{|c|c|c|c|}
\hline Measurements & Preoperative & Postoperative & p-value ${ }^{a}$ \\
\hline \multicolumn{4}{|l|}{ Hallux valgus angle } \\
\hline Mild $\left(<20^{\circ}\right)$ & $\mathrm{O}(0 \%)$ & $17(100 \%)$ & $<0.001$ \\
\hline Moderate ( 20 to $40^{\circ}$ ) & $4(23.6 \%)$ & $\mathrm{O}(0 \%)$ & \\
\hline Severe $\left(>40^{\circ}\right)$ & $13(76.4 \%)$ & $\mathrm{O}(0 \%)$ & \\
\hline \multicolumn{4}{|l|}{$\begin{array}{l}\text { Angle between the first } \\
\text { and second metatarsals }\end{array}$} \\
\hline Mild $\left(<11^{\circ}\right)$ & $2(11.8 \%)$ & $17(100 \%)$ & $<0.001$ \\
\hline Moderate (11 to $16^{\circ}$ ) & $13(76.4 \%)$ & $\mathrm{O}(0 \%)$ & \\
\hline Severe $\left(>16^{\circ}\right)$ & $2(11.8 \%)$ & $\mathrm{O}(0 \%)$ & \\
\hline
\end{tabular}



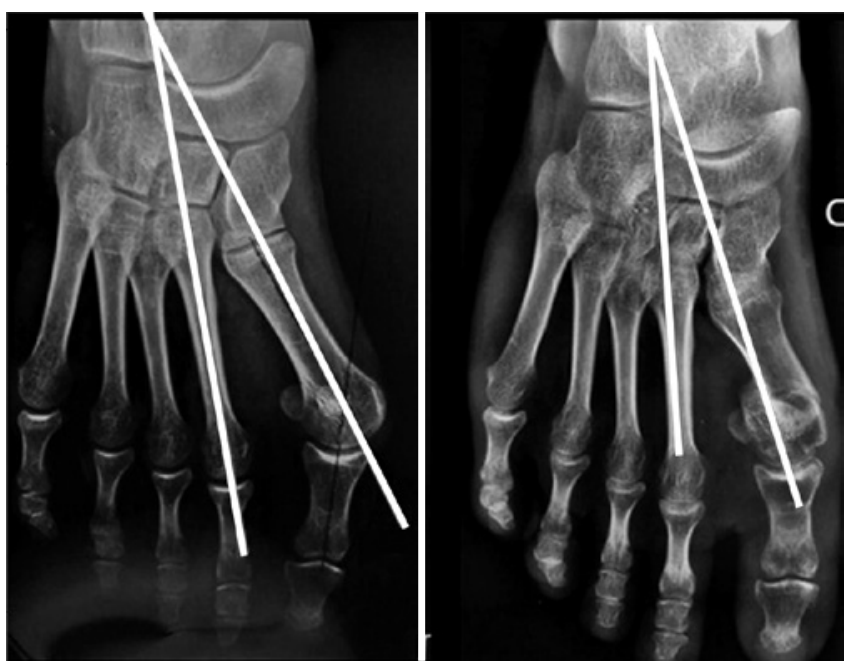

Figure 2. Anteroposterior X-ray showing correction of the intermetatarsal angle - Preoperative (left); and 12 months after surgery (right).

Table 3. Comparison of measurements of the hallux valgus angle and the angle between the first and second metatarsals, before and after intervention, in patients with hallux valgus treated with the PBS technique

\begin{tabular}{llccc} 
Measurements & Preoperative & Postoperative & $\begin{array}{c}\text { Mean reduction } \\
\text { during the } \\
\text { postoperative } \\
\text { period (95\%Cl) }\end{array}$ & p-value \\
\hline $\begin{array}{l}\text { Hallux valgus } \\
\text { angle (in } \\
\text { degrees) }\end{array}$ & $22.9 \pm 4.1$ & $7.8 \pm 2.7$ & $15.1(13.2$ to 17.0) & $<0.001$ \\
$\begin{array}{l}\text { Angle } \\
\text { between } \\
\text { the first }\end{array}$ & $13.0 \pm 2.2$ & $5.7 \pm 1.5$ & $7.3(5.9$ to 8.7$)$ & $<0.001$ \\
$\begin{array}{l}\text { and second } \\
\text { metatarsals } \\
\text { (in degrees) }\end{array}$ & & & & \\
\hline
\end{tabular}

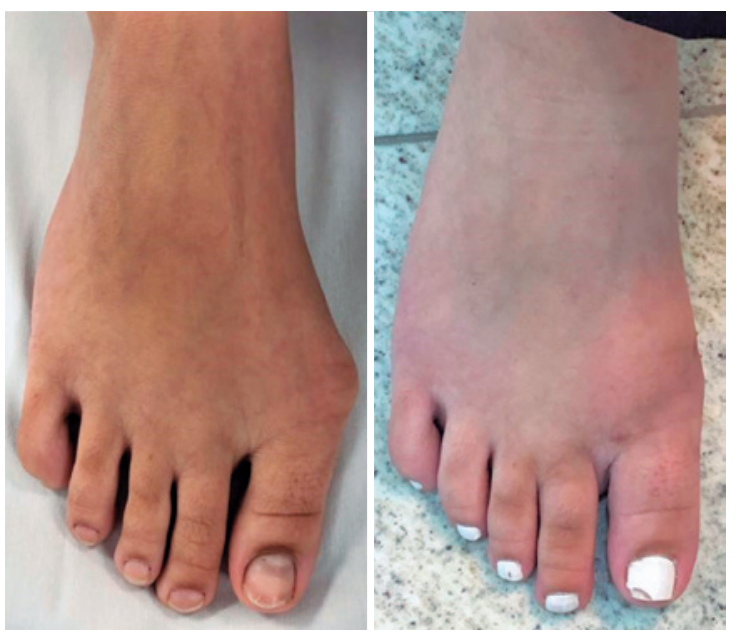

Figure 3. Images illustrating presence of hallux valgus (left); and the result 12 months after the surgical procedure (right).
Table 4 shows the effects of age on reduction of the HVA and IMA angles in this patient sample. The results demonstrated that age group had no statistically significant effect on angle reduction after execution of the PBS technique. It was observed that the mean HVA reduction among patients less than 60 years old was $16^{\circ}$ and the reduction among patients over the age of 60 was $13.9^{\circ}$, but this was not a significant difference $(p=0.246)$. With regard to the IMA, the reduction among patients less than 60 years old was $6.8^{\circ}$ and the reduction among patients over the age of 60 was $8^{\circ}$, which was also a non-significant difference $(p=0.365)$.

\section{Complications}

One patient had an IMA measuring $9^{\circ} 12$ months after surgery (classified as a mild deformity). Complications such as relapses to moderate or severe grades, postoperative infections, hallux varus, avascular necrosis, pseudoarthritis, or malunion of the first metatarsal were not observed in the radiographic assessments during the follow-up period.

\section{Discussion}

Over recent years, there has been an exponential increase in studies investigating percutaneous HV surgery. There is a growing body of evidence in the literature suggesting that percutaneous $\mathrm{HV}$ surgery is safe and reliable $\mathrm{e}^{(6)}$.

Table 4. Comparison of measurements of the hallux valgus angle and the angle between the first and second metatarsals, before and after intervention, in patients with hallux valgus treated with the PBS technique, stratified by age and side operated (laterality)

\begin{tabular}{|c|c|c|c|c|}
\hline Measurements & Preoperative & Postoperative & $\begin{array}{l}\text { Mean reduction } \\
\text { during the } \\
\text { postoperative } \\
\text { period }\end{array}$ & p-value ${ }^{\mathrm{a}}$ \\
\hline \multicolumn{5}{|c|}{ Hallux valgus angle (in degrees) } \\
\hline Age group & & & & 0.246 \\
\hline$<60$ years & $23.0 \pm 4.3$ & $7.0 \pm 2.3$ & 16.0 (13.7 to 18.3$)$ & \\
\hline $\begin{array}{l}60 \text { years or } \\
\text { over }\end{array}$ & $22.9 \pm 4.1$ & $9.0 \pm 3.0$ & 13.9 (10.1 to 17.6$)$ & \\
\hline Laterality & & & & 0.365 \\
\hline Right & $22.1 \pm 4.1$ & $7.8 \pm 3.2$ & 14.3 (11.8 to 16.9$)$ & \\
\hline Left & $23.9 \pm 4.1$ & $7.9 \pm 2.2$ & 16.0 (12.6 to 19.4$)$ & \\
\hline
\end{tabular}

\section{Angle between the first and second metatarsals (in degrees)}

Age group

0.390

\begin{tabular}{lllll}
$\begin{array}{l}<0 \text { years } \\
60 \text { years or }\end{array}$ & $12.7 \pm 2.2$ & $5.9 \pm 1.7$ & $6.8(4.6$ to 9.0$)$ & \\
$\begin{array}{l}\text { over } \\
\text { Laterality }\end{array}$ & & $5.4 \pm 1.3$ & $8.0(5.9$ to 10.1$)$ & \\
Right & $13.0 \pm 2.2$ & $6.0 \pm 1.5$ & $7.0(4.8$ to 9.2) & \\
Left & $13.0 \pm 2.3$ & $5.4 \pm 1.6$ & $7.6(5.4$ to 9.9) & \\
\hline
\end{tabular}

a Comparison of postoperative mean differences between groups. 
The PBS technique differs from other percutaneous techniques in terms of the characteristics of the first metatarsal osteotomy, which is complete and extra-articular, offering better protection of the blood supply to the head of the metatarsal(3). Although it does not involve use of the hardware generally used in other minimally invasive techniques, the PBS technique does demand use of radiation, with fluoroscopic images, to view correction of the deformity. Appropriate planning, an experienced surgeon, and correct technical execution are therefore essential to reduce exposure ${ }^{(12)}$.

In the PBS, the intrinsic stability of the osteotomy and the postoperative dressing are the two most important elements that maintain correction of the deformity.

This study demonstrates that the PBS technique was capable of correcting moderate and severe HV deformities, enabling correction of the two most important radiographic parameters: the hallux valgus angle and the intermetatarsal angle (HVA and IMA).

In 2016, Biz et al. ${ }^{(9)}$ assessed radiographic and functional results in patients with mild to severe HV who were treated with the Reverdin-Isham technique and Akin osteotomy, with a 48-month follow-up period. In that study, the HVA was reduced from $26.4^{\circ}$ to $12.3^{\circ}$ at the assessment 3 months after surgery, and to a mean of $13.9^{\circ}$ at the last follow-up assessment, while the IMA was reduced from $12.9^{\circ}$ to $9.0^{\circ}$.

In 2018, Liuni et al.(6) evaluated the PBS technique and observed better HVA and IMA correction, even in more severe deformities. Mean HVA reduced from $34^{\circ}$ to $9.3^{\circ}$ at 2 months and to $10.6^{\circ}$ at the 38 -month assessment, with an increase of just $1.3^{\circ}$ from the 2-month follow-up assessment to the last follow-up assessment. In turn, mean IMA reduced from $13.5^{\circ}$ to $8^{\circ}$ at the 2-month postoperative assessment and then to $8.5^{\circ}$ at the last follow-up assessment, with a $0.5^{\circ}$ correction loss from the 2-month follow-up assessment to the last follow-up assessment at 38 months.
The results observed in this study corroborate the literature and demonstrate adequate correction of the HVA and the IMA using the PBS technique. It was observed that radiographic angle correction was maintained up to 12 months after surgery, with no relapse to moderate or severe deformity.

The most important limitation of this study is the lack of a control group, which is an indispensable factor for comparison of results with those of traditional surgical techniques. The small number of patients is considered another limitation, since a larger cohort could have enabled stratification into groups to better analyze the efficacy of this technique. Additionally, the following radiographic parameters were not analyzed: measurements taken with a lateral view and weightbearing, the position of sesamoids in the AP view with weightbearing, and shortening of the first metatarsal in the AP view with weightbearing. There was also no analysis of clinical assessments of amplitude of metatarsophalangeal joint movement. The follow-up period of our series is relatively short (12 months), in particular for assessment of HV relapse.

\section{Conclusions}

The characteristics that should encourage use of the PBS technique are its minimally invasive character, the low incidence of complications, elimination of use of hardware for fixation, early mobilization with weightbearing, and minimal surgical scarring. According to the results presented, use of this technique achieved adequate radiographic correction of symptomatic HV classified as moderate to severe. The results were maintained up to the assessment performed 12 months after surgery. Notwithstanding, controlled and randomized studies with longer follow-up are needed to improve the recommendation level for the technique.

Authors' contributions: TMVS *(https://orcid.org/0000-0001-8764-8560) Conceived and planned the activities that gave rise to the study, wrote the article, participated in the review process, approved the final version; MVMGM *(https://orcid.org/0000-0002-7320-9628) Participated in the review process, approved the final version; JSG *(https://orcid.org/0000-0001-5996-6641) Participated in the review process, approved the final version; TEVM *(https://orcid.org/0000-0002-9162-5908) Participated in the review process, approved the final version; TBF *(https://orcid.org/O000-0002-61223609) Participated in the review process, approved the final version; MJTG *(https://orcid.org/0000-0003-4068-2598) Participated in the review process, approved the final version; MR *(https://orcid.org/0000-0001-7683-7470) Participated in the review process, approved the final version. All authors read and approved the final manuscript. ${ }^{*}$ ORCID (Open Researcher and Contributor ID) (iD).

\section{References}

1. Bösch P, Wanke S, Legenstein R. Hallux valgus correction by the method of Bösch: a new technique with a seven-to-ten-year follow-up. Foot Ankle Clin. 2000;5(3):485-98.

2. Nery CAS. Hallux valgus. Rev Bras Ortop. 2001;36(6):183-200.

3. Chan CX, Gan JZ, Chong HC, Rikhraj Singh I, Ng SYC, Koo K. Two year outcomes of minimally invasive hallux valgus surgery. Foot Ankle Surg. 2019;25(2):119-26.

4. Giannini S, Ceccarelli F, Bevoni R, Vannini F. Hallux valgus surgery: the minimally invasive bunion correction (SERI). Tech Foot Ankle Surg. 2003;2(1):11-20.
5. Magnan B, Pezzè L, Rossi N, Bartolozzi P. Percutaneous distal metatarsal osteotomy for correction of hallux valgus. J Bone Joint Surg Am. 2005;87(6):1191-9.

6. Liuni FM, Berni L, Fontanarosa A, Cepparulo R, Guardoli A, Pellegrini $A$, et al. Hallux valgus correction with a new percutaneous distal osteotomy: Surgical technique and medium term outcomes. Foot Ankle Surg. 2020;26(1):39-46.

7. Lucattelli G, Catani O, Sergio F, Cipollaro L, Maffulli N. Preliminary Experience With a Minimally Invasive Technique for Hallux Valgus Correction With No Fixation. Foot Ankle Int. 2020;41(1):37-43. 
8. Maffulli N, Longo UG, Marinozzi A, Denaro V. Hallux valgus: effectiveness and safety of minimally invasive surgery. A systematic review. Br Med Bull. 2011;97:149-67.

9. Biz C, Fosser M, Dalmau-Pastor M, Corradin M, Rodà MG, Aldegheri $\mathrm{R}$, et al. Functional and radiographic outcomes of hallux valgus correction by mini-invasive surgery with Reverdin-Isham and Akin percutaneous osteotomies: a longitudinal prospective study with a 48-month follow-up. J Orthop Surg Res. 2016;11(1):157.
10. Huang PJ, Lin YC, Fu YC, Yang YH, Cheng YM. Radiographic evaluation of minimally invasive distal metatarsal osteotomy for hallux valgus. Foot Ankle Int. 2011;32(5):S503-7.

11. Tanaka Y, Takakura Y, Takaoka T, Akiyama K, Fujii T, Tamai S. Radiographic analysis of hallux valgus in women on weightbearing and nonweightbearing. Clin Orthop Relat Res. 1997;(336):186-94.

12. Kay DB. Fixation Principles in Minimal Incision Hallux Valgus Surgery. Foot Ankle Clin. 2020;25(3):385-98. 